\title{
HUMAN SEMINAL PLASMA PROTEOME STUDY: A SEARCH FOR MALE INFERTILITY BIOMARKERS
}

\author{
Davalieva $\mathrm{K}^{1, *}$, Kiprijanovska $\mathrm{S}^{1}$, Noveski $\mathrm{P}^{1}$, Plaseski $\mathrm{T}^{2}$, Kocevska B ${ }^{2}$, \\ Plaseska-Karanfilska D ${ }^{1}$
}

*Corresponding Author: Dr. Katarina Davalieva, Research Centre for Genetic Engineering and Biotechnology "Georgi D. Efremov," Macedonian Academy of Sciences and Arts, Krste Misirkov 2, 1000 Skopje, Republic of Macedonia; Tel.: +389-2-3235-410; Fax: +389-2-3115-434; E-mail: katarina@manu.edu.mk

\section{ABSTRACT}

Seminal plasma is a potential source of biomarkers for many disorders of the male reproductive system including male infertility. Knowledge of the peptide and protein components of seminal fluid is accumulating especially with the appearance of highthroughput MS-based techniques. Of special interest in the field of male infertility biomarkers, is the identification and characterization of differentially expressed proteins in seminal plasma of men with normal and impaired spermatogenesis. However, the data obtained until now is still quite heterogeneous and with small percentage of overlap between independent studies. Extensive comparative analysis of seminal plasma proteome is still needed in order to establish a potential link between seminal plasma proteins and male infertility.

Keywords: Biomarkers, Male infertility, Mass spectrometry (MS), Matrix-assisted laser desorption/ionization time of flight (MALDI-TOF), Seminal plasma, Two-dimensional differential ingel electrophoresis (2-D DIGE), Two- dimensional polyacrylamide gel electrophoresis (2-D PAGE).

1 Research Centre for Genetic Engineering and Biotechnology "Georgi D. Efremov," Macedonian Academy of Sciences and Arts, 1000 Skopje, Republic of Macedonia

2 Endocrinology and Metabolic Disorders Clinic, Faculty of Medicine, Skopje, Republic of Macedonia

\section{INTRODUCTION}

The proteomes most likely to contain clinically useful disease biomarkers are those of human body fluids. Proteomics has raised great expectations for the discovery of biomarkers for improved diagnosis or stratification of a wide range of diseases [1]. Blood plasma and other body fluids, which also include seminal fluids, are expected to be excellent sources of protein biomarkers because they circulate through, or come in contact with a variety of tissues, and during this contact they are likely to pick up proteins secreted or shed by tissues, a hypothesis that has been tested and confirmed [2].

In addition to the general physiological importance of knowing the composition of seminal fluid, medical interest centers on two main areas: infertility and prostate cancer. Male infertility is a widespread medical condition with large societal and emotional costs. Between one in six and one in 10 couples seek medical help for the problem of sub fertility. In $20.0-25.0 \%$ of cases the infertility problem is due to the male partner, in $30.0-40.0 \%$ the problem is predominantly female; in approximately $30.0 \%$ of cases, abnormalities are found in both partners, and in $15.0 \%$ no specific factor is identified [3]. Since seminal fluid has an important role in spermatozoa survival and overall fertilization success, its impairment can be directly connected to infertility. 
Seminal plasma contains many distinct protein components that are important for the functioning and survival of spermatozoa. It is a mixture of secretions from several male accessory glands, including prostate, seminal vesicles, epididymis, and Cowper's gland. The average protein concentration of human seminal plasma ranges from 35 to $55 \mathrm{~g} / \mathrm{L}$ making it a rich, as well as an easily accessible source for protein identification. Seminal plasma has the feature common to many other body fluids, namely it is characterized by a high dynamic range of protein abundance, making low-abundance components difficult to analyze.

Characterization of Human Seminal Plasma Protein and Peptide Constituents. Research of seminal plasma proteome began using two-dimensional polyacrylamide gel electrophoresis (2-D PAGE) and the electrophoretic transfer of proteins to nitrocellulose sheets followed by subsequent detection with immunological procedures in 1981 [4]. By this approach, a few seminal plasma proteins such as prostatic acid phosphatase (PAP), some glycoproteins and creatinin kinases, were identified. Two-dimensional (2-D) gels coupled with mass spectrometry (MS) were applied to study the role of seminal plasma proteins in impaired spermatogenesis in 2001, and about 750 spots were detected in the 2-D maps of seminal plasma from a fertile men [5]. However, only two seminal plasma constituents, namely, PAP and prostatic specific antigen (PSA) were identified by matrix-assisted laser desorption/ ionization time of flight (MALDI-TOF) in this study, as the two major spot clusters in the 2-D map of the seminal plasma proteome. By using 2-D PAGE with MALDI and liquid chromatographyelectrospray ionization (LC-ESI), Fung and colleagues [6] identified 100 different protein constituents in seminal plasma. This study established that there were multiple post-translational variants of the majority of the proteins. Overall, hormones, growth factors and bio-active peptides were detected and identified. Pilch and Mann [7] successfully catalogued 932 proteins in seminal plasma using Fourier transform MS and two consecutive stages of MS fragmentation. This study identified proteins known to be characteristic for each of the organs contributing to the formation of seminal plasma: prostate, seminal vesicles, epididymis, and the bulbourethral gland. The large proportions of the identified pro- teins were extracellular, secreted by the male accessory glands as well as extracellular matrix proteins that are required for the classical functions of seminal fluid. The second class of the identified proteins was found to originate from prostasomes, while the third class of proteins was the result of an epithelial shredding. The overall numbers and proportions of proteins in the identified proteome indicated that the predominant functions are in clot formation and liquefaction, in metabolic support and protection for the spermatozoa and in immunological reactions. The identification of seminal plasma protein and peptide constituents by using combined 2-D PAGE/ MS/MS and 2D nano high performance liquid chromatography (HPLC)/MS/MS is currently in progress at our institution.

Identification of some well defined subgroup of human seminal plasma proteins such as heparin-binding proteins that are included in the fertilization process were also carried out by the use of affinity chromatography, 2D electrophoresis and MALDI-TOF MS [8]. Difference in the expression of sperm membrane proteins between fertile and infertile males [9], as well as proteins associated with human fertilization capability [10], have also been investigated.

Comparative Analysis of Seminal Plasma Proteome With Identification of Potential Infertility Biomarkers. The research in the field of comparative proteomic analysis of male infertilityassociated seminal plasma proteins has been carried out for almost a decade. However, extensive comparative analysis of seminal plasma proteome in order to establish a potential link between seminal plasma proteins and male infertility is still lacking.

By investigating the differential expression of proteins between two or more biologically different states of reduced male fertility or infertility, potential diagnostic or prognostic markers may be identified. The gold standard techniques for this is still 2-D PAGE/2-D DIGE coupled to MS because this approach is amenable to the separation and visualization of a wide range of proteins, together with their post translational variants.

Identification of 61 differentially expressed proteins based on 2-D PAGE and tandem MS analysis of seminal plasma of azoospermic and vasectomized patients has been reported by Sarita-Garibaldi and coworkers [11]. Two- dimensional DIGE combined with MS was also carried out to find seminal plasma 
proteins associated specifically with azoospermia and revealed a total of four potential markers for non obstructive azoospermia [12]. The identified potential biomarkers were stabilin 2 (STAB2), $135 \mathrm{kd}$ centrosomal protein (CP135), guanine nucleotide, releasing protein (GNRP), and prolactin-inducible protein (PIP). In a recent study, we utilized the 2-D DIGE/MS approach to detect differential protein expression of seminal plasma proteins between four distinct groups of men with normozoospermia, oligozoospermia, asthenozoospermia and azoospermia [13]. The results from our study demonstrated that normozoospermic, oligozoospermic and asthenozoospermic groups have similar seminal plasma protein profiles, resulting in no statistically significant differences in protein expression. However, in the azoospermic group there were a total of eight proteins with a statistically significant increase of expression in comparison with the rest of the studied groups. From the eight differentially expressed proteins, seven were successfully identified by MS as fibronectin (FINC), prostatic acid phosphatase (PAP), proteasome subunit $\alpha$ type-3 (PSA3), $\beta-2-$ microglobulin (B2MG), galectin-3 binding protein (LG3BP), prolactin inducible protein (PIP) and cytosolic non specific dipeptidase (CNDP2). All of the reported proteins have already been reported as constituents of seminal plasma. Most of these proteins (FINC, PAP, B2MG, LG3BP, PIP) are localized in the extracellular region and have the molecular function of protein binding. From all the differentially expressed spots found in this study, PAP was found to be exclusively increased in azoospermic patients compared with the rest of the studied groups. Prostatic acid phosphatase is the most abundant phosphatase in human prostate tissue and has been studied extensively, primarily due to its clinical relevance as a biomarker of prostate carcinoma [14]. Several other studies have implicated PAP in male infertility before, with enzyme levels inversely correlated with sperm concentration [15-17].

Another study, based on the comparative analysis of the levels of PIP in fertile and infertile men, has proposed that PIP could be an immunoglobulin G-binding protein [18]. Prolactin inducible protein was shown to exist in several isoforms in seminal plasma by Western blot. The pattern of PIP isoform variability in seminal plasma from fertile and infertile men is quite complex but one multimeric form of PIP was confirmed to be absent from the seminal plasma of fertile men.

The levels of fibronectin (FINC fragments in seminal plasma samples were found higher in seminal plasma with abnormal semen characteristics than in the normozoospermic group [19]. The results suggest that seminal plasma FINC fragments may contribute to fertilization and the analysis of FINC fragmentation may have a diagnostic value in andrological investigations.

Proteomic analysis of seminal plasma from asthenozoospermia patients revealed a rich source of biomarker candidates for male infertility, proposing that functional abnormalities of the epididymis and prostate can contribute to asthenozoospermia [20]. Among the identified proteins, DJ-1, a protein that has been shown to be involved in the control of oxidative stress, was down regulated in asthenozoospermia patients and was proposed as a candidate biomarker for this condition.

In a conclusion, the research in the field of seminal plasma proteome and the search for biomarkers of male infertility is still ongoing. An in-depth understanding of the seminal plasma proteome would contribute greatly to the elucidation of the roles of seminal plasma proteins in the regulation of motility and to the establishment of biomarkers for male infertility. There have been different sets of proteins proposed as biomarkers in different conditions of reduced fertility and/or infertility. However, only small set of proteins such as PAP, PIP, FINC are found differentially expressed in male infertility by independent studies. One of the reasons for this very low percentage of overlap between independent studies is that different proteomics techniques and their combinations were used. Therefore, the data from these studies is quite heterogeneous. However, the appearance of high-throughput MSbased techniques allows more detailed investigation of the proteomes of interest, among which is human seminal plasma proteome, and holds promise on more reproducible results in the future.

\section{ACKNOWLEDGMENTS}

The proteomic study of seminal plasma performed was supported by grant CRP/MAC0901 from the International Centre for Genetics Engineering and Biotechnology, Trieste, Italy (to 
D. Plaseska-Karanfilska). We are grateful to Cedric Broussard for the mass spectrometry analysis that were performed at the Plate-forme Proteomique Paris 5 (3P5), Institut Cochin, Inserm U1016 UMR8104 and Université Paris Descartes Sorbonne Paris Cité, Paris, France. We are also grateful to Dr. Martina Marchetti-Deschmann for the training in Peptide Mass Fingerprinting that was performed during the stay of Dr. Katarina Davalieva at the Institute of Chemical Technologies and Analytics, Division: Instrumental Analytical Chemistry, Vienna University of Technology, Austria. Dr. Katarina Davalieva's 2-week stay was supported by the FP7 project No. 229458 from the European Commission.

\section{REFERENCES}

1. Etzioni R, Kooperberg C, Pepe M, Smith R, Gann $\mathrm{PH}$. Combining biomarkers to detect disease with application to prostate cancer. Biostatistics. 2003; 4(4): 523-538.

2. Zhang H, Liu AY, Loriaux P, et al. Mass spectrometric detection of tissue proteins in plasma. Mol Cell Proteomics. 2007; 6(1): 64-71.

3. De Kretser DM, Baker HW. Infertility in men: recent advances and continuing controversies. J Clin Endocrinol Metab. 1999; 84(10): 3443-3450.

4. Edwards JJ, Tollaksen SL, Anderson NG. Proteins of human semen. I. Two-dimensional mapping of human seminal fluid. Clin Chem. 1981; 27(8): 13351340.

5. Starita-Geribaldi M, Poggioli S, Zucchini M, et al. Mapping of seminal plasma proteins by two-dimensional gel electrophoresis in men with normal and impaired spermatogenesis. Mol Hum Reprod. 2001; 7(8): 715-722.

6. Fung KY, Glode LM, Green S, Duncan MW. A comprehensive characterization of the peptide and protein constituents of human seminal fluid. Prostate. 2004; 61(2): 171-181.

7. Pilch B, Mann M. Large-scale and high-confidence proteomic analysis of human seminal plasma. Genome Biol. 2006; 7(5): R40.

8. Kumar V, Hassan MI, Tomar AK, et al. Proteomic analysis of heparin-binding proteins from human seminal plasma: a step towards identification of molecular markers of male fertility. J Biosci. 2009; 34(6): 899-908.
9. Rajeev SK, Reddy KVR. Sperm membrane protein profiles of fertile and infertile men: identification and characterization of fertility associated sperm antigen. Hum Reprod. 2004; 19(2): 234-242.

10. Zhang J, Wu J, Huo R, et al. ERp57 is a potential biomarker for human fertilization capability. Mol Hum Reprod. 2007; 13(9): 633-639.

11. Starita-Geribaldi M, Roux F, Garin J, Chevallier D, Fenichel P, Pointis G. Development of narrow immobilized $\mathrm{pH}$ gradients covering one $\mathrm{pH}$ unit for human seminal plasma proteomic analysis. Proteomics. 2003; 3(8): 1611-1619.

12. Yamakawa K, Yoshida K, Nishikawa H, Kato T, Iwamoto T. Comparative analysis of interindividual variations in the seminal plasma proteome of fertile men with identification of potential markers for azoospermia in infertile patients. J Androl. 2007; 28(6): 858-865.

13. Davalieva K, Kiprijanovska S, Noveski $\mathrm{P}$, et al. Proteomic analysis of seminal plasma in men with different spermatogenic impairment. Andrologia. 2012; 44(4): 256-264.

14. Azumi N, Traweek ST, Battifora H. Prostatic acid phosphatase in carcinoid tumors. Immunohistochemical and immunoblot studies. Am J Surg Pathol. 1991; 15(8): 785-790.

15. Dave BN, Rindani TH. Acid phosphatase activity in human semen. Int J Fertil. 1988; 33(1): 45-47.

16. Vaubourdolle M, Clavel JP, Gonzales J, Galli A. Evaluation of acid phosphatase isoenzymes in seminal fluid from normozoospermic, oligozoospermic, azoospermic and asthenoteratozoospermic men. Andrologia. 1985; 17(6): 598-604.

17. Ziyyat A, Barraud-Lange V, Sifer C, Ducot B, Wolf JP, Soufir JC. Paradoxical increase of sperm motility and seminal carnitine associated with moderate leukocytospermia in infertile patients. Fertil Steril. 2008; 90(6): 2257-2263.

18. Chiu WW, Chamley LW. Human seminal plasma prolactin-inducible protein is an immunoglobulin G-binding protein. J Reprod Immunol. 2003; 60(2): 97-111.

19. Katnik-Prastowska I, Przybysz M, Chelmonska-Soyta A. Fibronectin fragments in human seminal plasma. Acta Biochim Pol. 2005; 52(2): 557-560.

20. Wang J, Zhang HR, Shi HJ, et al. Proteomic analysis of seminal plasma from asthenozoospermia patients reveals proteins that affect oxidative stress responses and semen quality. Asian J Androl. 2009; 11(4): 484-491. 